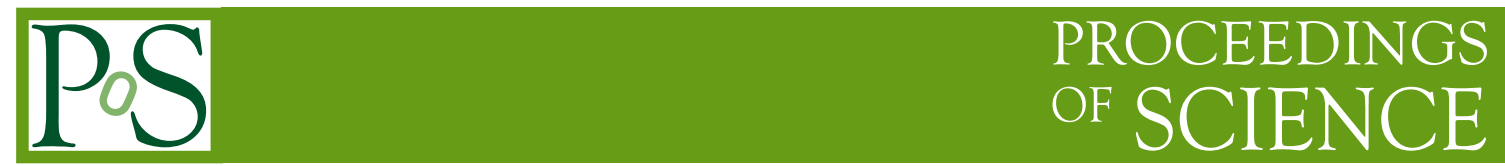

\title{
Heavy meson interquark potential
}

\author{
V. Vento* \\ Departamento de Física Teórica -IFIC, Universidad de Valencia-CSIC, E-46100 Burjassot \\ (Valencia), Spain. \\ E-mail: vicente.vento@uv.es

\section{P. González and V. Mathieu} \\ Departamento de Física Teórica-IFIC, Universidad de Valencia-CSIC, E-46100 Burjassot \\ (Valencia), Spain.
}

\begin{abstract}
The resolution of Dyson-Schwinger equations leads to the freezing of the QCD running coupling (effective charge) in the infrared, which is best understood as a dynamical generation of a gluon mass function, giving rise to a momentum dependence which is free from infrared divergences. We calculate the interquark static potential for heavy mesons by assuming that it is given by a massive One Gluon Exchange interaction and compare with phenomenologyical fits inspired by lattice QCD. We apply these potential forms to the description of quarkonia and conclude that, even though some aspects of the confinement mechanism are absent in the Dyson-Schwinger formalism, the spectrum can be reasonably reproduced. We discuss possible explanations for this outcome.
\end{abstract}

International Workshop on QCD Green's Functions, Confinement and Phenomenology, September 05-09, 2011

Trento Italy

${ }^{*}$ Speaker. 


\section{Introduction}

Understanding low-energy properties of Quantum Chromodynamics (QCD) represents a theoretical challenge. The development of nonperturbative techniques are essential in this regime. Lattice gauge theory $[1,2]$ constitutes a nonperturbative regularisation scheme which allows numerical solutions of the theory describing properties of interacting QCD matter [3]. The accuracy of lattice results has been tremendously improved during the past decade with the availability of more powerful computers $[4,5]$, and lattice results are considered in many instances the data which other nonperturbative schemes should reproduce.

The approximate resolution of the Dyson-Schwinger (DS) equations is another nonperturbative approach which has progressed considerably in the last ten years in great part due to the interplay between their findings and lattice results. It is a more analytical approach and has led to a very appealing physical picture establishing that the QCD running coupling (effective charge) freezes in the deep infrared. This property can be best understood from the point of view of a dynamical gluon mass generation $[6,7]$.

The aim of this presentation is to investigate the consequences associated to the static interaction one can derive from this picture. For this purpose we calculate numerically the one gluon exchange (OGE) static potential deriving from the DS equations and we compare it to phenomenological potentials whose shape has been inspired by lattice computations. The application of these potentials to the description of quarkonia is discussed.

\section{Heavy quark dynamics from lattice}

For sufficiently heavy quarks, it can be shown in lattice QCD, that the bound state problem becomes essentially nonrelativistic and the dynamics is controlled approximately by a Schrödinger equation with a static potential. Lattice calculations, without dynamical quarks (quenched), give rise to a static potential of the form $[8,9]$

$$
V(r)=-a / r+b r
$$

containing the perturbative expected Coulomb term plus an additional linear term.

The Cornell group, prior to the lattice QCD derivation, using $a$ and $b$ as parameters, successfully applied this potential to the phenomenological description of the low lying quarkonia states $[10,11,12,13]$ and therefore this potential function is known as the Cornell potential.

Another static interquark potential shape has been guessed by performing unquenched lattice QCD calculations. Using Kogut-Susskind fermions and adopting the lattice spacing from the $\rho$ mass measurements a potential with the following structure,

$$
V(r)=(-\alpha / r+\beta r)\left(\frac{1-e^{-\gamma r}}{\gamma r}\right)
$$

has been shown to describe these lattice results[14]. This parameterization shows screening, associated to quarks loops, which has been cast in the form of the additional factor multiplying the Cornell potential, leading at large values of $r$ to a constant $\beta / \gamma$. Henceforth we shall call this shape the Aachen potential and we shall choose its parameters to provide a reasonable fit to the spectrum. 


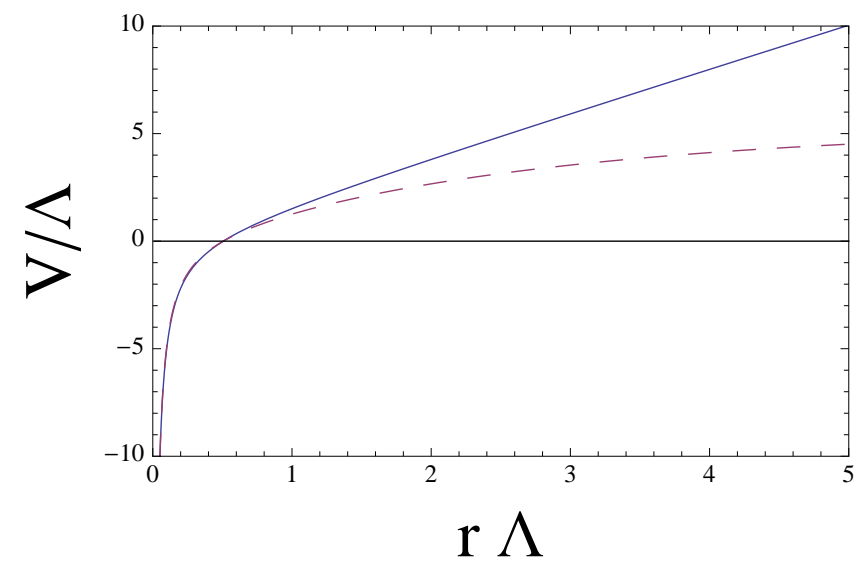

Figure 1: We show the two potentials described in the text : Cornell (solid) and Aachen (dashed). For the Cornell and Aachen potentials the parameters have been chosen phenomenologically to provide a reasonable description of the spectrum and their values are given in Sec. 4.

We show the potentials in Fig. 1 for comparison ${ }^{1}$. We use in what follows these potentials in our discussion since they emphasize the two aspects we want to discuss, linear confinement and screening.

\section{Heavy quark dynamics from Dyson-Schwinger Equations}

Infrared finite solutions for the gluon propagator of quenched QCD are obtained from the gauge-invariant nonlinear Dyson-Schwinger equations formulated in the Feynman gauge of the background field method. These solutions may be fitted using a massive propagator [6,7]. Even though the gluon is massless at the level of the fundamental QCD Lagrangian, and remains massless to all order in perturbation theory, the nonperturbative QCD dynamics generates an effective, momentum-dependent mass, without affecting the local $S U(3)_{c}$ invariance, which remains intact.

The gluon mass generation is a purely nonperturbative effect associated with the existence of infrared finite solutions for the gluon propagator, $\Delta\left(q^{2}\right)$, i.e. solutions with $\Delta^{-1}(0)>0$. Such solutions may be fitted by a "massive" euclidean propagator of the form $[6,16]$

$$
\Delta\left(q^{2}\right)=\frac{1}{q^{2}+m^{2}\left(q^{2}\right)}
$$

where $m^{2}\left(q^{2}\right)$ depends nontrivially on the momentum transfer $q^{2}$. One physically motivated possibility, which we shall use in here, is the so called logarithmic mass running, which is defined by

$$
m^{2}\left(q^{2}\right)=m_{0}^{2}\left[\ln \left(\frac{q^{2}+\rho m_{0}^{2}}{\Lambda^{2}}\right) / \ln \left(\frac{\rho m_{0}^{2}}{\Lambda^{2}}\right)\right]^{-1-\delta},
$$

\footnotetext{
${ }^{1}$ The spin-dependent corrections to the potentials above can also be derived from lattice QCD [15]. We do not consider them here.
} 
where $m_{0}, \rho$ and $\delta$ are parameters whose values are chosen to fit the lattice propagator and $\Lambda$ is the $Q C D$ scale. Note that in the limit $q^{2} \rightarrow 0$ one obtains $m^{2}(0)=m_{0}^{2}$, giving meaning to $m_{0}$.

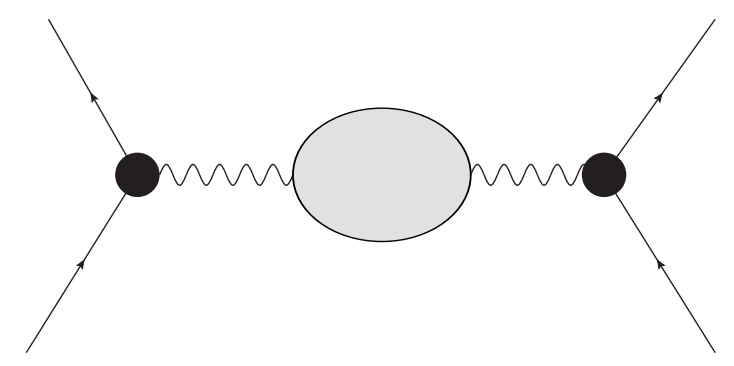

Figure 2: One gluon exchange contribution to the potential.

Because of the presence of this dynamical gluon mass the strong effective charge extracted from these solutions freezes at a finite value, giving rise to an infrared fixed point for QCD [6, 7]. This nonperturbative generalization of $\alpha\left(q^{2}\right)$, the QCD running coupling, comes in the form

$$
a\left(q^{2}\right)=\left[\beta_{0} \ln \left(\frac{q^{2}+\rho m^{2}\left(q^{2}\right)}{\Lambda^{2}}\right)\right]^{-1},
$$

where $a=\frac{\alpha}{4 \pi}$ and we take $\beta_{0}=11-2 n_{f} / 3$ where $n_{f}$ is the number of flavors. Note that its zero gluon mass limit leads to the LO perturbative coupling constant momentum dependence. The $m\left(q^{2}\right)$ in the argument of the logarithm tames the Landau pole, and $a\left(q^{2}\right)$ freezes at a finite value in the IR, namely $a^{-1}(0)=\beta_{0} \ln \left(\rho m^{2}(0) / \Lambda^{2}\right)$.

We have constructed a simple potential model where the main source of dynamics is the One Gluon Exchange potential (see Fig. 2) with the propagator and coupling defined by Eqs. 3.1, 3.2 and 3.3 [17]. In Fig. 3 we show the potential derived from the DS equations for the following range of parameters: $m_{0} \sim 360-480 \mathrm{MeV}, \rho=1-4, \delta=1 / 11[6,18,19]$. The value of $\Lambda$ has been taken to be $300 \mathrm{MeV}$. In order to adjust the behavior at the origin to the data we have used $\beta_{0}$ corresponding to 4 flavors. We have removed from this calculation the additive infinite self-energy contribution associated with the static sources [8], a procedure we call Sommer subtraction. In lattice QCD this is done normalizing the potential such that $V\left(r_{0}\right)=0$ where $r_{0}$ is the Sommer scale [20]. We proceed in the same way but take the subtraction point at the zero of the phenomenological potentials, which happens to be at $r_{0} \sim 0.35 \mathrm{fm}$ (see next section). We call the result of this construction the Dyson-Schwinger (DS) potential [17]. We obtain a potential which resembles the Aachen and not the Cornell potential.

It should be noted that the shape of the DS potential does not vary considerably when we change the parameters within the expected theoretical range. There is no way to reproduce the large $r$ behavior of the Cornell potential, instead the DS potential flattens and becomes asymptotically constant, similarly to the screened potentials. However, it should not! The approximations used to find the solution to the DS equations do not contain quark loops and therefore they incorporate no mechanism for screening, i.e. a mechanism derivable from the breaking of the string [14, 21]. The truncation in the DS set of equations and the absence of multigluon exchanges might be responsible for the missing linear rise at large $r$. 


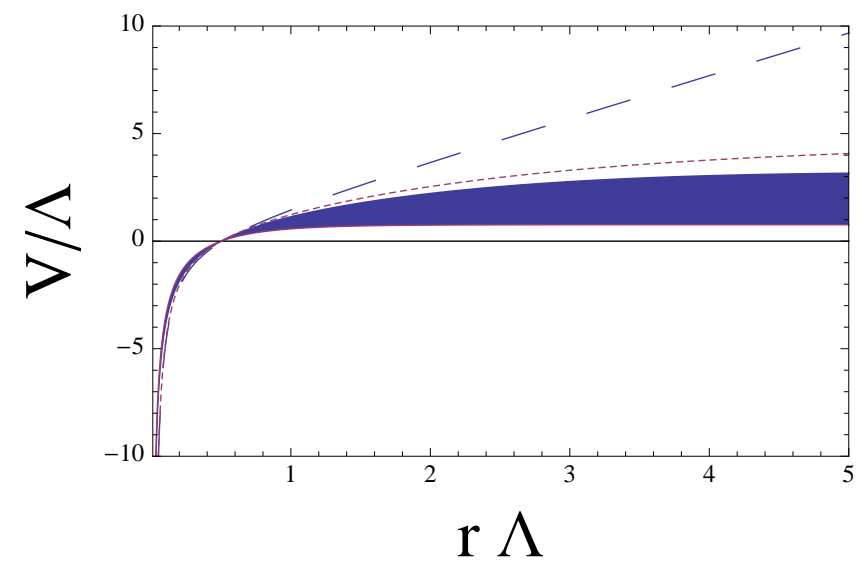

Figure 3: We show the DS potential after the Sommer subtraction, for $n_{f}=4$. The shaded range corresponds to $m_{0} \sim 360-480 \mathrm{MeV}$ and $\rho \sim 1-4$. For comparison we plot the Cornell (dashed) and Aachen (dotted) potentials.

\section{Quarkonia description by static potentials}

The Cornell potential was introduced for phenomenological reasons predating the complete lattice derivation. From the spin-averaged quarkonia spectra it was evident that the underlying potential could not be purely Coulomb type [10] . Therefore, the potential was implemented by a sum of the perturbative expectation plus an additional linear term, recall Eq. 2.1. Aiming at a universal treatment for charmonium $(c \bar{c})$ and bottomonium $(b \bar{b})$ the Cornell potential has been used with the same values of the parameters, $a$ and $b$, in both cases, for the quark masses, $m_{c}$ and $m_{b}$, respectively. A typical range of values providing a reasonable fit to the masses of the low lying states (up to $1.0 \mathrm{GeV}$ excitation energy) is $a \sim 0.51-0.52$ and $\sqrt{b} \sim 412-427 \mathrm{MeV}$ $[11,12,13]$. An example of such spectral fit for charmonium is provided in Table 1 where we have chosen $\sqrt{b}=427 \mathrm{MeV}$ and $a=0.52$ with $m_{c}=1350 \mathrm{MeV}$ and compared the results with masses of experimental resonances having well established $J^{P C}$ quantum numbers, most of them with $J^{P C}=1^{--}$coming from ISR (Initial State Radiation) processes. The Cornell model provides a good fit to the lower states (at most $30 \mathrm{MeV}$ difference with data) but it cannot accommodate all the known higher energy resonances but only some of them (we use charmonium instead of bottomonium to clarify the effect). For instance $\psi(4040), \psi(4160)$ and $\psi(4415)$ maybe assigned to the $3 s, 2 d$ and $4 s$ states respectively. Then other two resonances, cataloged in the Particle Data Group Review [22] as $X(4260)$ and $X(4360)$, cannot be fitted.

The screened potentials introduced initially to fit lattice data have been used phenomenologically $[23,24]$. However, the applicability of screened potentials to the spectral description has been a matter of debate [25] since their use is not justified above the meson-meson string breaking threshold. We assume that in the case of quarkonia there is an effective string breaking threshold sufficiently high in energy, to allow for a description of the known spectrum in terms of screened potentials. For the sake of comparison the results from the Aachen potential for $m_{c}=1400 \mathrm{MeV}$ and the same values for the corresponding Cornell parameters $\alpha=a, \beta=b$ are presented in Table 1. The value of $\gamma=0.38 \mathrm{fm}^{-1}$ is fixed to get a reasonable spectral fit. It is important to emphasize 


\begin{tabular}{|cccc|c|}
\hline$n_{r} L$ & $M_{\text {Cornell }}$ & $M_{\text {Aachen }}$ & $M_{P D G}$ & $M_{D S}$ \\
\hline & $\mathrm{MeV}$ & $\mathrm{MeV}$ & $\mathrm{MeV}$ & $\mathrm{MeV}$ \\
\hline $1 s$ & 3069 & 3143 & $3096.916 \pm 0.011$ & 3151 \\
$2 s$ & 3688 & 3665 & $3686.09 \pm 0.04$ & 3660 \\
$1 d$ & 3806 & 3775 & $3772.92 \pm 0.35$ & 3761 \\
$3 s$ & 4147 & 4001 & $4039 \pm 1$ & 4004 \\
$2 d$ & 4228 & 4072 & $4153 \pm 3$ & 4070 \\
$4 s$ & 4539 & 4255 & $4263_{-9}^{+8}$ & 4273 \\
$3 d$ & 4601 & 4306 & $4361 \pm 9 \pm 9$ & 4321 \\
$5 s$ & 4829 & 4564 & $4421 \pm 4$ & 4487 \\
$4 d$ & 4879 & 4609 & & 4526 \\
$6 s$ & 5218 & 4629 & $4664 \pm 11 \pm 5$ & 4651 \\
\hline \hline $5 d$ & 5260 & 4663 & & 4718 \\
\hline \hline $1 p$ & 3502 & 3527 & $3525.3 \pm 0.2$ & 3515 \\
$2 p$ & 3983 & 3894 & & 3886 \\
\hline
\end{tabular}

Table 1: Calculated charmonium masses, $M_{\text {Cornell }}, M_{\text {Aachen }}$ and $M_{D S}$ from the Cornell, Aachen and DS potentials. For Cornell and Aachen $a=\alpha=0.52$ and $\sqrt{b}=\sqrt{\beta}=427 \mathrm{MeV}$. The remaining parameter in the Aachen potential has been chosen to be $\gamma=0.38 \mathrm{fm}^{-1}$. For DS $\rho=1$ and $m_{0}=345.7 \mathrm{MeV}$. The charm masses are $m_{c}=1350 \mathrm{MeV}$ for Cornell and $m_{c}=1400 \mathrm{MeV}$ for Aachen and DS. Masses for experimental candidates, $M_{P D G}$, have been taken from [22]. For $p$ waves we quote the centroid of the $n p_{0}, n p_{1}$ and $n p_{2}$ states.

that the values of the parameters of the Aachen potential extracted from lattice data [14] can only give a reasonable description of the masses of the low lying quarkonia.

One should keep in mind that these potentials do not contain spin-dependent terms which makes them reliable only when these terms do not play a major role. We use here the conventional approximations, e.g. we consider that these potentials should fit better the spin triplet states and take the centroids of $p$ states as data for comparison with our results.

The main difference between the Cornell and Aachen potentials refers to the description of the higher excited states. The Aachen potential may allow a one to one assignment of the calculated states to the data.

The similarity of the DS potential (for certain parameter sets) to the Aachen potential as shown in Fig. 3 motivates the exercise of fitting the spectrum with the DS potential. As can bee seen in Table 1 this can be achieved for a set of parameters which is close to the expected theoretical range. A low value of the mass, $m_{0}=345.7 \mathrm{MeV}$, is necessary since only with a value close to $\Lambda$ one gets sufficient strength to achieve, after Sommer subtraction, an asymptotic behavior close to the Aachen potential used in our spectral fit.

Let us discuss the limitations of the parameters used in the fit. The $\beta_{0}$ expression is fixed by QCD. $\beta_{0}$ is the leading order coefficient of the beta function of the theory, which is scheme independent; the small difference in value implied by the choice of $n_{f}$ has almost no effect on the results. $\Lambda$ is a scale fixed by dimensional transmutation. We take $300 \mathrm{MeV}$ and keep it fixed. The 
values obtained in experimental fits range from $250-300 \mathrm{MeV}$ but we have not even used that liberty. The $\delta$ value and the variations in the values of $\rho$ and $m_{0}$ are within the limits provided by the DS calculations, in particular $\Lambda<m_{0}<2 \Lambda[10,21]$.

\section{Discussion and Conclusions}

In order to interpret the above results we have to resort again to lattice calculations. In ref. [26] the authors investigated the origin of the long range linearly rising potential. Using a mechanism described in ref. [27] they were able to subtract the contribution of the center vortices leading to a flat potential as shown in Fig. 4 on the left. On the right of Fig. 4 we show the DS potential after Sommer subtraction and the linear rise of the Cornell potential. It is quite apparent that the figure resembles the one on the left. Therefore we may tentatively conclude that the DS potential contains the physics associated with the approximate gluon interaction but does not contain the physics of the confinement mechanism. We do not advocate any mechanism for confinement in QCD, we only adhere to the fact that the confinement mechanism, whatever it be, is the mechanism behind the rising potential in the quenched approximation.
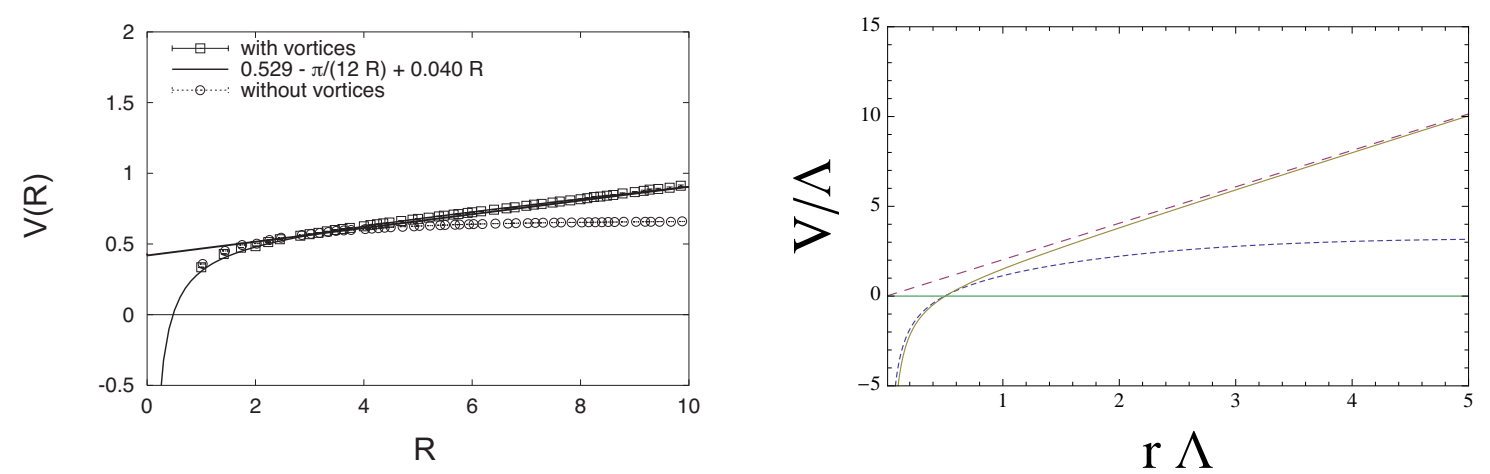

Figure 4: The figure on the left corresponds to the calculation of ref.[26]. In the figure on the right the dotted line corresponds to the DS potential for $n_{f}=4$ and $m_{0}=360 \mathrm{MeV}$ and $\rho=1$. The dashed line corresponds to the linear piece of the Cornell potential. The full Cornell potential is drawn (solid line) for comparison.

A surprising result of our calculation is the actual similarity of the DS potential to the Aachen potential as shown in Fig. 3. The latter arises due to the breaking of the string and is represented by a screened potential [14]. The similarity is astonishing more so since we have used conventional values for $\rho=1, \delta=1 / 11, \Lambda=300 \mathrm{MeV}$ and only varied $m_{0}$, which becomes for the best fit $m_{0}=345.7 \mathrm{MeV}$ within the expected range $\Lambda<m_{0}<2 \Lambda[7,16]$.

Not aiming at such precision if we fit the upper extreme of the shaded region, where the DS potential is defined by $m_{0}=360 \mathrm{MeV}$ and $\rho=1$, to the Aachen potential shape function we get $\alpha=0.40, \sqrt{\beta}=412 \mathrm{MeV}$ and $\gamma=0.53 \mathrm{fm}^{-1}$. The other extreme set of parameters for the DS potential, the lower edge of the shaded region, $m_{0}=480 \mathrm{MeV}$ and $\rho=4$, leads to $\alpha=0.37, \sqrt{\beta}=361 \mathrm{MeV}$ and $\gamma=1.81 \mathrm{fm}^{-1}$. It is clear then, that the main difference between the parametrizations arises due to the value of the different screening ranges, which in the Aachen potential is controlled by $\gamma$. 
Is this similarity accidental or does string breaking imply a dilution of the confinement mechanism associated with multigluon exchanges and/or higher order truncation schemes? Does the Sommer subtraction introduce the energy scale of string breaking ? More research needs to be done in order to understand the confinement and string breaking mechanisms though our investigation hints a possible scenario.

In conclusion, we have calculated the OGE potential associated to the approximate resolution of the Dyson-Schwinger equations for the gluon propagator. The low $r$ behavior is determined perturbatively. The large $r$ behavior is certainly nonperturbative. The Sommer procedure, to avoid self-energy effects of the static charges, leads to a potential which is not negative at large $r$. The approximate resolution of the DS equations is not able to reproduce, at the level of the OGE, the Cornell potential. The DS equations together with the Sommer subtraction is close to the Aachen potential that contains a string breaking mechanism. The fact that we have to push the parameters to the limit of the allowed region to reproduce the spectrum maybe due to the fact that we have not treated the quark mass terms appropriately.

The potential derived from the DS equations might contain most of the dynamics associated with the interquark interaction apart from a nontrivial constant which might be related, in the unquenched calculation, to the confinement mechanism.

\section{Acknowledgement}

We would like to thank very instructive conversations with Arlene Aguilar, Daniele Binosi and Joannis Papavassiliou. VV would like to acknowledge clarifying conversations with Stefan Olejnik regarding ref. [26], with Nora Brambilla regarding pNRQCD and the static lattice potential and with Simonetta Liuti about the consequences of our results. We would like tho thank the organizers of the Workshop for the invitation to present these results and the ECT* for local support. This work has been supported by HadronPhysics2, by MICINN (Spain) grant FPA2010-21750-C02-01, AIC10-D-000598 and by GVPrometeo2009/129. VM has been supported by a post-doctoral grant from CPAN and by a contract from HadronPhysics2.

\section{References}

[1] K. G. Wilson, Phys. Rev. D 10 (1974) 2445.

[2] M. Creutz, Phys. Rev. D 21 (1980) 2308.

[3] M. Creutz, arXiv:1103.3304 [hep-lat].

[4] C. Sachrajda, PoS LATTICE2010 (2010) 018 [arXiv:1103.5959 [hep-lat]].

[5] P. Hagler, Prog. Theor. Phys. Suppl. 187 (2011) 221.

[6] J. M. Cornwall, Phys. Rev. D 26, 1453 (1982).

[7] A. C. Aguilar and J. Papavassiliou, JHEP 0612, 012 (2006)

[8] G. S. Bali, Phys. Rept. 343 (2001) 1-136. [hep-ph/0001312].

[9] N. Brambilla, A. Pineda, J. Soto and A. Vairo, Rev. Mod. Phys. 77 (2005) 1423 [arXiv:hep-ph/0410047]. 
[10] E. Eichten, K. Gottfried, T. Kinoshita, J. B. Kogut, K. D. Lane, T. -M. Yan, Phys. Rev. Lett. 34 (1975) 369-372.

[11] C. Quigg, J. L. Rosner, Phys. Rept. 56, 167-235 (1979).

[12] E. Eichten, K. Gottfried, T. Kinoshita, K. D. Lane, T. -M. Yan, Phys. Rev. D21 (1980) 203.

[13] E. Eichten, S. Godfrey, H. Mahlke and J. L. Rosner, Rev. Mod. Phys. 80 (2008) 1161 [arXiv:hep-ph/0701208].

[14] K. D. Born, E. Laermann, N. Pirch, T. F. Walsh, P. M. Zerwas, Phys. Rev. D40 (1989) 1653-1663.

[15] Y. Koma and M. Koma, Nucl. Phys. B 769 (2007) 79 [arXiv:hep-lat/0609078].

[16] A. C. Aguilar, D. Binosi and J. Papavassiliou, Phys. Rev. D 78, 025010 (2008).

[17] P. Gonzalez, V. Mathieu, V. Vento, [arXiv:1108.2347 [hep-ph]].

[18] A. C. Aguilar and J. Papavassiliou, Eur. Phys. J. A 35, 189 (2008).

[19] A. C. Aguilar, D. Binosi, J. Papavassiliou and J. Rodriguez-Quintero, Phys. Rev. D 80, 085018 (2009);

[20] R. Sommer, Nucl. Phys. B 411 (1994) 839 [arXiv:hep-lat/9310022].

[21] S. Kratochvila, P. de Forcrand, Nucl. Phys. B671 (2003) 103-132. [hep-lat/0306011].

[22] K. Nakamura et al. [Particle Data Group], J. Phys. G 37 (2010) 075021.

[23] Y. B. Ding, K. T. Chao and D. H. Qin, Phys. Rev. D 51 (1995) 5064 [arXiv:hep-ph/9502409].

[24] P. Gonzalez, A. Valcarce, H. Garcilazo and J. Vijande, Phys. Rev. D 68 (2003) 034007 [arXiv:hep-ph/0307310].

[25] E. S. Swanson, J. Phys. G 31 (2005) 845 [arXiv:hep-ph/0504097].

[26] J. Greensite, S. Olejnik, Phys. Rev. D67 (2003) 094503. [hep-lat/0302018].

[27] P. de Forcrand, M. D’Elia, Phys. Rev. Lett. 82 (1999) 4582-4585. [hep-lat/9901020]. 